\title{
Impact of Metabolic Syndrome on Subclinical Atherosclerosis in Asymptomatic Individuals
}

Gyung-Min Park, MD; Hyonggin An, PhD; Seung-Whan Lee, MD; Young-Rak Cho, MD;

Eun Ha Gil; Sung Ho Her, MD; Hyun Woo Park, MD; Jung-Min Ahn, MD; Duk-Woo Park, MD;

Soo-Jin Kang, MD; Young-Hak Kim, MD; Cheol Whan Lee, MD; Dong Hyun Yang, MD;

Joon-Won Kang, MD; Tae-Hwan Lim, MD; Hong-Kyu Kim, MD; Jaewon Choe, MD;

Seong-Wook Park, MD; Seung-Jung Park, MD

Background: Little is known about subclinical atherosclerosis on coronary computed tomographic angiography (CCTA) in asymptomatic individuals with metabolic syndrome (MetS).

Methods and Results: We analyzed 5,213 asymptomatic individuals who underwent CCTA. A cardiac event was defined as a composite of all-cause death, myocardial infarction, unstable angina, or coronary revascularization. Of the study participants, 2,042 (39.2\%) had MetS. MetS was an independent predictor of significant coronary artery disease $(C A D)$ in at least 1 coronary artery (odds ratio $[O R]=1.992,95 \%$ confidence interval $[\mathrm{Cl}]=1.623-2.445$, $\mathrm{P}<0.001)$ and significant $C A D$ in the left main $(L M)$ or proximal left anterior descending $(L A D)$ artery $(O R=2.151,95 \%$ $\mathrm{Cl}=1.523-3.037, \mathrm{P}<0.001$ ). During the follow-up period (median 28.1 [interquartile range, 19.2-36.5] months), 111 individuals had 114 cardiac events. Individuals with MetS were significantly associated with more cardiac events than those without ( $\mathrm{RR}$ [rate ratio] $=1.67,95 \% \mathrm{Cl}=1.15-2.43, \mathrm{P}=0.007$ ). In the MetS group, individuals with significant $\mathrm{CAD}$ had the majority of cardiac events $(\mathrm{RR}=64.33,95 \% \mathrm{Cl}=29.17-141.88, \mathrm{P}<0.001)$. Furthermore, in the MetS with significant CAD group, those with significant CAD in the LM or proximal LAD had more cardiac events $(R R=2.63$, $95 \% \mathrm{Cl}=1.51-4.59, \mathrm{P}=0.001)$.

Conclusions: MetS was associated with subclinical atherosclerosis on CCTA with subsequent high risk for cardiac events. These findings suggest the importance of reducing unfavorable metabolic conditions in asymptomatic individuals. (Circ J 2015; 79: 1799-1806)

Key Words: Atherosclerosis; Coronary artery disease; Metabolic syndrome

$\mathbf{O}$ ver the past decades, the prevalence of metabolic syndrome (MetS), characterized by a cluster of conditions including abdominal obesity, glucose intolerance, hypertension, and dyslipidemia, has rapidly increased in most countries of the world. ${ }^{1,2}$ MetS is a strong risk factor for cardiovascular mortality and morbidity. ${ }^{3-5}$ Furthermore, even in asymptomatic individuals, MetS is associated with a more than $50 \%$ increase in the risk of sudden death, independent of coronary artery disease (CAD) risk factors. ${ }^{6}$ Therefore, MetS has become a major public health concern. ${ }^{7}$
Recently, with the advent of multidetector row computed tomography, coronary computed tomography angiography (CCTA) has proven effective in providing comprehensive information on CAD, including lesion location, severity, and characteristics of atherosclerotic plaque. ${ }^{8}$ In previous studies of asymptomatic individuals undergoing CCTA, the effect of MetS on the risk of subclinical atherosclerosis was studied. However, those studies focused on the prevalence of subclinical atherosclerosis and plaque characteristics, so the clinical implications of these findings were lacking. ${ }^{7,9}$ Therefore, through

Received October 31, 2014; revised manuscript received March 18, 2015; accepted March 26, 2015; released online May 8, 2015 Time for primary review: 26 days

Department of Cardiology, Daejeon St. Mary's Hospital, College of Medicine, The Catholic University of Korea, Daejeon (G.-M.P., E.H.G., S.H.H.); Department of Biostatistics, College of Medicine, Korea University, Seoul (H.A.); Department of Cardiology (S.-W.L., J.-M.A., D.-W.P., S.-J.K., Y.-H.K., C.W.L., S.-W.P., S.-J.P.), Department of Radiology (D.H.Y., J.-W.K., T.-H.L.), The Health Screening and Promotion Center (H.-K.K., J.C.), Asan Medical Center, University of Ulsan College of Medicine, Seoul; Department of Cardiology, Dong-A University Hospital, Busan (Y.-R.C); and Department of Cardiology, Soon Chun Hyang University Hospital Bucheon, Bucheon (H.W.P.), Korea

The first two authors contributed equally to this article (G.-M.P., H.A.).

Mailing address: Seung-Whan Lee, MD, PhD, Department of Cardiology, Asan Medical Center, University of Ulsan College of Medicine, 388-1 Poongnap-dong, Songpa-gu, Seoul 138-736, Korea. E-mail: seungwlee@amc.seoul.kr

ISSN-1346-9843 doi:10.1253/circj.CJ-14-1197

All rights are reserved to the Japanese Circulation Society. For permissions, please e-mail: cj@j-circ.or.jp 


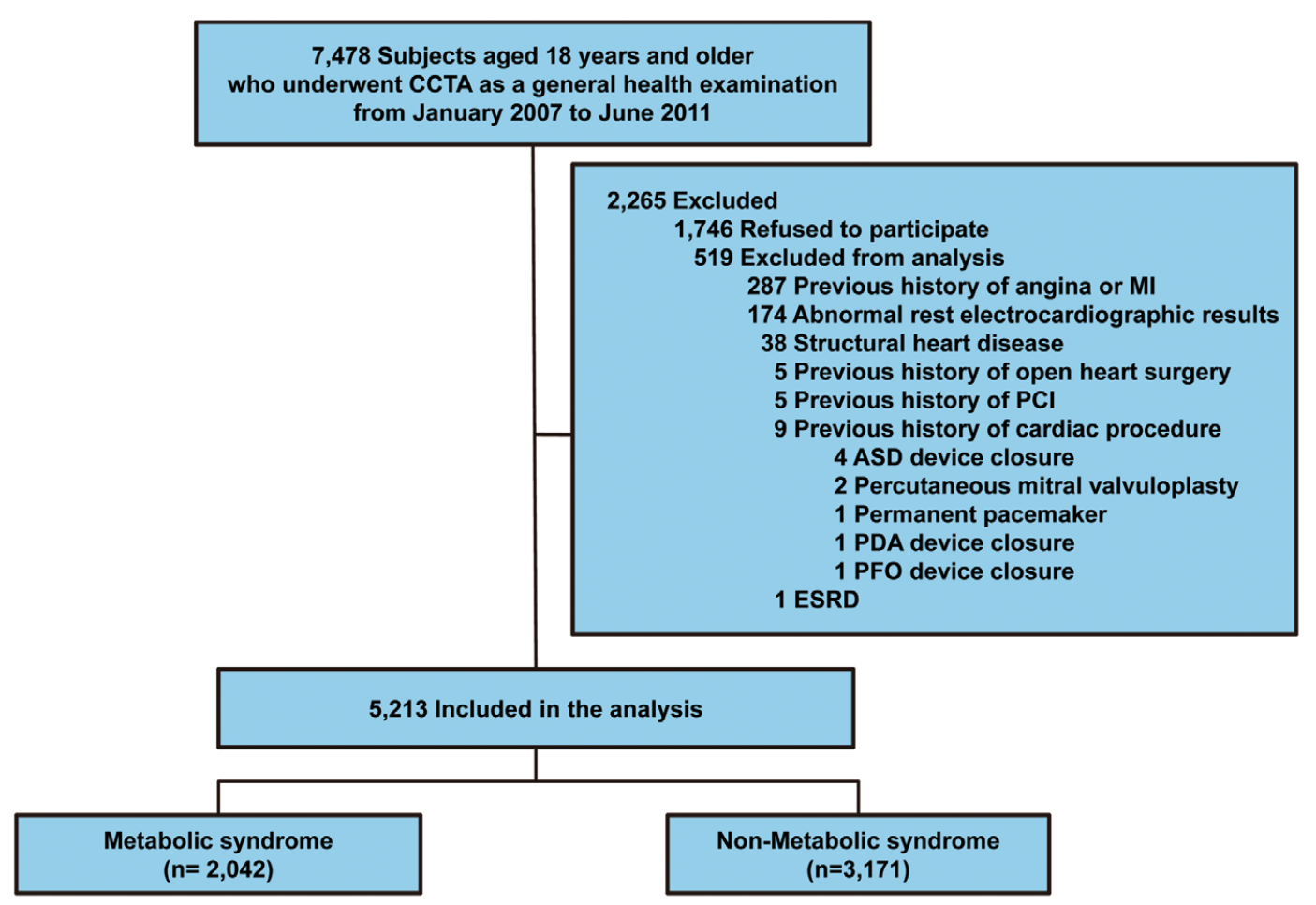

Figure 1. Overview of the study population. ASD, atrial septal defect; CCTA, coronary computed tomographic angiography; ESRD, end stage renal disease; MI, myocardial infarction; PCI, percutaneous coronary intervention; PDA, patent ductus arteriosus; PFO, patent foramen ovale.

a large cohort of asymptomatic individuals undergoing CCTA, we sought to investigate the effect of MetS on the risk of subclinical atherosclerosis and clinical outcome.

\section{Methods}

\section{Study Population}

From January 2007 to June 2011, 7,478 consecutive South Korean individuals aged 18 years and older, who had undergone self-referral CCTA evaluation as part of a general health examination in the Health Screening and Promotion Center at the Asan Medical Center, were enrolled. All subjects were made aware of the possible risks associated with CCTA and provided informed consent. Of these, 5,732 (76.7\%) consented to participate this study. Subjects with (1) previous history of angina or myocardial infarction (MI), (2) abnormal rest ECG results (ie, pathological Q waves, ischemic ST segment or T wave changes, or left bundle-branch block), (3) structural heart disease, (4) prior history of open heart surgery, (5) past history of percutaneous coronary intervention (PCI), (6) previous cardiac procedure, and (7) renal insufficiency (creatinine $>1.5 \mathrm{mg} / \mathrm{dl}$ ) were excluded. Thus, 5,213 subjects were finally enrolled and analyzed (Figure 1). This study was approved by the local Institutional Review Board at the Asan Medical Center, Seoul, Korea. All patients provided written informed consent.

The basic demographic data of the subjects were acquired from a database maintained by the Health Screening and Promotion Center at the Asan Medical Center. Any medical history of angina, MI, stroke, structural heart disease, open heart surgery, PCI, previous cardiac procedure, diabetes mellitus, hypertension, or hyperlipidemia; family history of CAD and smoking status were collected from a systemized questionnaire administered prior to the general health examination. In the general health examination, height, body weight, body mass index, waist circumference, and blood pressure were measured, and an ECG was performed. Moreover, fasting plasma glucose, glycated hemoglobin, uric acid, blood urea nitrogen, creatinine, total cholesterol, high-density and low-density lipoprotein cholesterol, triglycerides, and high-sensitivity C-reactive protein concentrations were measured on the day of the examination after a fasting period of $\geq 12 \mathrm{~h}$. CAD risk was calculated using the Framingham risk model. ${ }^{10}$

\section{Definition of MetS}

MetS was defined according to the 2005 revision of the criteria from the National Cholesterol Education Program Adult Treatment Panel III. ${ }^{11}$ Abdominal obesity was redefined on the basis of an Asian-specific cut-off point, as recommended in the National Cholesterol Education Program Adult Treatment Panel III criteria. Subjects were considered to have MetS if they had 3 or more of the following abnormalities: abdominal obesity (waist circumference $\geq 90 \mathrm{~cm}$ in men and $\geq 80 \mathrm{~cm}$ in women), hypertriglyceridemia $\geq 150 \mathrm{mg} / \mathrm{dl}$, low high-density lipoprotein cholesterol $(<40 \mathrm{mg} / \mathrm{dl}$ in men and $<50 \mathrm{mg} / \mathrm{dl}$ in women), high blood pressure $(\geq 130 / 85 \mathrm{mmHg}$ ) or use of antihypertensive medication, and high fasting glucose $(\geq 100 \mathrm{mg} / \mathrm{dl})$ or use of antidiabetic medication. 


\section{CCTA Image Acquisition and Analysis}

CCTA was conducted using either a single-source 64-slice CT (LightSpeed VCT, GE, Milwaukee, WI, USA) or dual-source CT (Somatom Definition, Siemens, Erlangen, Germany). Patients with no contraindication to $\beta$-adrenergic blocking agents and with initial heart rates greater than 65 beats/min received an oral dose of $2.5 \mathrm{mg}$ bisoprolol (Concor, Merck, Darmstadt, Germany) $1 \mathrm{~h}$ before the CT examination. CT scanning was performed in the prospective ECG-triggering mode or the retrospective ECG-gating mode with ECG-based tube current modulation. Two puffs $(2.5 \mathrm{mg})$ of isosorbidedinitrate (Isoket spray, Schwarz Pharma, Monheim, Germany) were sprayed into the patient's mouth before contrast injection. During CCTA acquisition, $60-80 \mathrm{ml}$ of iodinated contrast (Iomeron 400 , Bracco, Milan, Italy) was injected at $4 \mathrm{ml} / \mathrm{s}$, followed by a 40-ml saline flush. A region of interest was placed on the ascending aorta, and image acquisition was automatically initiated once a selected threshold (100 Hounsfield units [HU]) had been reached using bolus tracking. A standard scanning protocol was used, and the tube voltage and tube current-time product were adjusted according to the patient's body size as follows: $100 \mathrm{kVp}$ or $120 \mathrm{kVp}$ tube voltage; $240-400 \mathrm{mAs}$ per rotation (dual-source CT); and 400-800 mA (64-slice CT) tube current.

All CCTA scans were analyzed using a dedicated workstation (Advantage Workstation, GE; or Volume Wizard, Siemens) by experienced cardiovascular radiologists (D.H.Y., T.-H.L. and J.-W.K.) blinded to the clinical information. Final decisions regarding the findings were reached by consensus. According to the guidelines of the Society of Cardiovascular Computed Tomography, a 16-segment coronary artery tree model was used. ${ }^{12}$ A coronary artery calcium score (CACS) was measured as described, ${ }^{13}$ with categorization by scores of $0,1-10,11-100,101-400$, and $>400 .{ }^{14}$ Plaques were defined as structures $>1 \mathrm{~mm}^{2}$ within and/or adjacent to the vessel lumen, which could be clearly distinguished from the lumen and surrounding pericardial tissue. Plaques containing calcified tissue involving more than $50 \%$ of the plaque area (density $>130 \mathrm{HU}$ ) were classified as calcified, plaques with $<50 \%$ calcium were classified as mixed, and plaques without calcium were classified as non-calcified lesions. ${ }^{15}$ The contrast-enhanced portion of the coronary lumen was semi-automatically traced at the site of maximal stenosis and compared with the mean value of the proximal and distal reference sites. ${ }^{16}$ Stenosis $\geq 50 \%$ was defined as significant. In addition, overall plaque burden was determined from coronary artery plaque scores, calculated from modified Duke prognostic scores, segment stenosis scores, and segment involvement scores, as described. ${ }^{17}$

\section{Clinical Outcomes}

Follow-up clinical data were obtained by a review of medical records at the end of August 2012. Cardiac events were defined as a composite of all-cause death, non-fatal MI, unstable angina requiring hospitalization, or coronary revascularization. Death was identified by identification numbers, which were assigned to the subjects on their birth certificates, in the National Statistical Office. ${ }^{18}$ The diagnosis of MI was based on the presence of new $\mathrm{Q}$ waves in at least 2 contiguous leads or an elevation of creatine kinase or its MB isoenzyme to at least 3-fold the upper limit of the normal range at follow-up. Revascularization was performed if there were stenosis of at least $50 \%$ of the diameter on invasive coronary angiography with a positive stress test or if there was a stenosis of at least $70 \%$ on invasive coronary angiography. ${ }^{19}$

\section{Statistical Analysis}

Categorical data were compared with chi-square statistics or Fisher's exact test and presented as frequencies. Continuous variables were analyzed using unpaired Student's t test and presented as mean \pm standard deviation. Multivariate logistic regression analyses were performed to identify clinical predictors of significant CAD in at least 1 coronary artery and significant CAD in the left main (LM) or left anterior descending (LAD) artery on CCTA. A backward elimination process was used to develop the final multivariable model, and an adjusted odds ratio (OR) with $95 \%$ confidence intervals (CI) was calculated. For clinical outcomes, we calculated the incidence rate per 1,000 person-years as the number of cases divided by the 1,000 person-years. That is, the incidence rate=number of cases/the summation of time spent in the study across all participants $\times 1,000$. CIs of the incidence rates were also estimated according to the assumption that the number of cases followed a Poisson distribution. All reported P-values are 2 -sided, and $\mathrm{P}<0.05$ was considered statistically significant. Data manipulation and statistical analyses were conducted using SAS $^{\circledR}$ Version 9.1 (SAS Institute Inc, Cary, NC, USA).

\section{Results}

\section{Baseline Characteristics of the Patients}

The baseline characteristics of the study population according to MetS are listed in Table 1. The mean age of the study population was $53.9 \pm 8.3$ years and 3,810 $(73.1 \%)$ participants were male. The average Framingham risk score was $7.9 \pm 5.4 \%$. Of the study population, 2,042 (39.2\%) individuals had MetS. Individuals with MetS had more comorbid conditions than those without MetS.

\section{CCTA Findings}

Table 2 shows the CCTA findings of individuals with and without MetS. Individuals with MetS had a higher CACS than those without $(\mathrm{P}<0.001)$. The incidence of any coronary atherosclerotic, calcified, non-calcified, or mixed plaque was significantly higher in individuals with $\mathrm{MetS}(\mathrm{P}<0.001$ for all $)$. Individuals with MetS also had higher plaque burden scores including the modified Duke prognostic score, segment stenosis score, and segment involvement score $(\mathrm{P}<0.001$ for all). Individuals with MetS had more significant $\mathrm{CAD}$, multivessel disease, and significant CAD in the LM or proximal LAD on CCTA than those without $(\mathrm{P}<0.001$ for all).

After adjustment for clinical risk factors, multivariate logistic regression analysis revealed that MetS was an independent predictor for significant $\mathrm{CAD}$ in at least 1 coronary artery $(\mathrm{OR}=1.992,95 \% \mathrm{CI}=1.623-2.445, \mathrm{P}<0.001)$ and significant $\mathrm{CAD}$ in the LM or proximal LAD on CCTA $(\mathrm{OR}=2.151,95 \%$ $\mathrm{CI}=1.523-3.037, \mathrm{P}<0.001$ ) (Table 3).

\section{Clinical Outcomes}

During the follow-up period (median 28.1 [interquartile range, 19.2-36.5] months), a total of 114 cardiac events occurred in 111 individuals: 19 deaths, 2 non-fatal MIs, 2 unstable angina requiring hospitalization, and 91 coronary revascularizations (Table 4). Individuals with MetS was significantly associated with more cardiac events than those without (RR [rate ratio] $=1.67,95 \% \mathrm{CI}=1.15-2.43, \mathrm{P}=0.007)$. However, there was no significant difference in the hard events such as death, $\mathrm{MI}$ and unstable angina between individuals with and without MetS $(\mathrm{RR}=1.01,95 \% \mathrm{CI}=0.44-2.33, \mathrm{P}=0.984)$. In the MetS group, those with significant $\mathrm{CAD}$ had more cardiac events than those without $(\mathrm{RR}=64.33$, 95\% CI=29.17-141.88, 
Table 1. Baseline Characteristics of Asymptomatic Individuals With and Without MetS

\begin{tabular}{|c|c|c|c|c|}
\hline & $\begin{array}{c}\text { Overall } \\
(n=5,213)\end{array}$ & $\begin{array}{c}\text { MetS } \\
(n=2,042)\end{array}$ & $\begin{array}{l}\text { Non-MetS } \\
(n=3,171)\end{array}$ & $P$ value \\
\hline Age, years & $53.9 \pm 8.3$ & $55.1 \pm 8.3$ & $53.1 \pm 8.2$ & $<0.001$ \\
\hline Male, n (\%) & $3,810(73.1)$ & $1,571(76.9)$ & $2,289(70.6)$ & $<0.001$ \\
\hline Body mass index, $\mathrm{kg} / \mathrm{m}^{2}$ & $24.7 \pm 2.9$ & $26.2 \pm 2.8$ & $23.7 \pm 2.6$ & $<0.001$ \\
\hline Waist circumference, $\mathrm{cm}$ & $86.1 \pm 8.4$ & $90.7 \pm 7.6$ & $83.2 \pm 7.5$ & $<0.001$ \\
\hline Systolic BP, mmHg & $119.6 \pm 12.9$ & $123.9 \pm 12.9$ & $116.8 \pm 12.1$ & $<0.001$ \\
\hline Diastolic BP, mmHg & $76.3 \pm 10.3$ & $79.4 \pm 10.1$ & $74.3 \pm 10.0$ & $<0.001$ \\
\hline Diabetes mellitus, $\mathrm{n}(\%)$ & $843(16.2)$ & $544(26.6)$ & $299(9.4)$ & $<0.001$ \\
\hline Hypertension, n (\%) & $1,920(36.8)$ & $1,239(60.7)$ & $681(21.5)$ & $<0.001$ \\
\hline Hyperlipidemia, n (\%) & $1,646(31.6)$ & $1,049(51.4)$ & $597(18.8)$ & $<0.001$ \\
\hline Current smoker, n (\%) & $1,250(24.0)$ & $553(27.1)$ & $697(22.0)$ & $<0.001$ \\
\hline Family history of CAD, ${ }^{*} \mathrm{n}(\%)$ & $807(15.5)$ & $330(16.2)$ & $477(15.0)$ & 0.276 \\
\hline Total cholesterol, mg/dl & $195.2 \pm 34.4$ & $192.7 \pm 37.0$ & $196.8 \pm 32.4$ & $<0.001$ \\
\hline LDL-cholesterol, mg/dl & $121.3 \pm 30.1$ & $119.4 \pm 32.6$ & $122.4 \pm 28.3$ & $<0.001$ \\
\hline HDL-cholesterol, mg/dl & $53.0 \pm 13.5$ & $47.7 \pm 11.7$ & $56.4 \pm 13.4$ & $<0.001$ \\
\hline Triglyceride, mg/dl & $134.3 \pm 83.6$ & $173.6 \pm 96.7$ & $108.9 \pm 61.8$ & $<0.001$ \\
\hline Fasting blood glucose, $\mathrm{mg} / \mathrm{dl}$ & $105.3 \pm 21.4$ & $113.4 \pm 24.9$ & $100.1 \pm 16.9$ & $<0.001$ \\
\hline Glycated hemoglobin, \% & $5.7 \pm 0.8$ & $6.0 \pm 0.9$ & $5.5 \pm 0.7$ & $<0.001$ \\
\hline Creatinine, $\mathrm{mg} / \mathrm{dl}$ & $0.9 \pm 0.2$ & $0.9 \pm 0.2$ & $0.9 \pm 0.2$ & $<0.001$ \\
\hline Uric acid, mg/dl & $5.6 \pm 1.4$ & $5.9 \pm 1.5$ & $5.4 \pm 1.3$ & $<0.001$ \\
\hline $\mathrm{hsCRP} \geq 2 \mathrm{mg} / \mathrm{dl}, \%$ & $52(1.0)$ & $16(0.8)$ & $36(1.1)$ & 0.212 \\
\hline Framingham risk score & $7.9 \pm 5.4$ & $10.1 \pm 5.7$ & $6.5 \pm 4.7$ & $<0.001$ \\
\hline
\end{tabular}

Values are given as mean \pm standard deviation or number (\%). ${ }^{*} \mathrm{CAD}$ in a first-degree relative of any age. BP, blood pressure; CAD, coronary artery disease; HDL, high-density lipoprotein; hsCRP, high-sensitivity C-reactive protein; LDL, low-density lipoprotein; MetS, metabolic syndrome.

\begin{tabular}{|c|c|c|c|c|}
\hline CCTA characteristics & $\begin{array}{l}\text { Overall } \\
(n=5,213)\end{array}$ & $\begin{array}{c}\text { MetS } \\
(n=2,042)\end{array}$ & $\begin{array}{l}\text { Non-MetS } \\
(n=3,171)\end{array}$ & $P$ value \\
\hline Mean CACS & $44.0 \pm 154.7$ & $67.1 \pm 200.6$ & $29.1 \pm 113.6$ & $<0.001$ \\
\hline CACS score classification, $\mathrm{n}(\%)$ & & & & $<0.001$ \\
\hline 0 & 3,352 (64.4) & $1,099(53.9)$ & 2,253 (71.1) & \\
\hline $1-10$ & $482(9.3)$ & $220(10.8)$ & $262(8.3)$ & \\
\hline $11-100$ & $831(16.0)$ & $398(19.5)$ & $433(13.7)$ & \\
\hline $101-400$ & $406(7.8)$ & $238(11.7)$ & $168(5.3)$ & \\
\hline$>400$ & 134 (2.6) & $83(4.1)$ & $51(1.6)$ & \\
\hline Any plaque, n (\%) & $2,244(43.0)$ & $1,101(53.9)$ & $1,143(36.0)$ & $<0.001$ \\
\hline \multicolumn{5}{|l|}{ Plaque characteristics, n (\%) } \\
\hline Calcified plaque & 1,596 (30.6) & 815 (39.9) & $781(24.6)$ & $<0.001$ \\
\hline Non-calcified plaque & $956(18.3)$ & $474(23.2)$ & $482(15.2)$ & $<0.001$ \\
\hline Mixed plaque & $479(9.2)$ & $256(12.5)$ & $223(7.0)$ & $<0.001$ \\
\hline Modified Duke prognostic index & $1.2 \pm 0.7$ & $1.3 \pm 0.8$ & $1.1 \pm 0.6$ & $<0.001$ \\
\hline Segment stenosis score & $0.7 \pm 2.0$ & $1.0 \pm 2.6$ & $0.5 \pm 1.6$ & $<0.001$ \\
\hline Segment involvement score & $1.1 \pm 1.8$ & $1.5 \pm 2.1$ & $0.9 \pm 1.6$ & $<0.001$ \\
\hline \multicolumn{5}{|l|}{ Stenosed vessels, n (\%) } \\
\hline Significant CAD & $440(8.4)$ & $251(12.3)$ & $189(6.0)$ & $<0.001$ \\
\hline 1-vessel disease & $320(6.1)$ & $180(8.8)$ & $140(4.1)$ & $<0.001$ \\
\hline Multivessel disease & $120(2.3)$ & $71(3.5)$ & $49(1.5)$ & $<0.001$ \\
\hline $\begin{array}{l}\text { Significant CAD in the LM or } \\
\text { proximal LAD }\end{array}$ & $144(2.8)$ & $87(4.3)$ & $57(1.8)$ & $<0.001$ \\
\hline
\end{tabular}

Values are given as mean \pm standard deviation or number (\%). CACS, coronary artery calcium score; CCTA, coronary computed tomographic angiography; LAD, left anterior descending artery; LM, left main. Other abbreviations as in Table 1. 


\begin{tabular}{|c|c|c|c|c|c|c|c|c|c|c|c|c|}
\hline & \multicolumn{3}{|c|}{ Univariate analysis (1) } & \multicolumn{3}{|c|}{ Multivariate analysis $(1)^{\star}$} & \multicolumn{3}{|c|}{ Univariate analysis (2) } & \multicolumn{3}{|c|}{ Multivariate analysis $(2)^{\star \star}$} \\
\hline & OR & $95 \% \mathrm{Cl}$ & $P$ value & OR & $95 \% \mathrm{Cl}$ & $P$ value & OR & $95 \% \mathrm{Cl}$ & $P$ value & OR & $95 \% \mathrm{Cl}$ & $P$ value \\
\hline Age, years & 1.089 & $\begin{array}{c}1.077- \\
1.102\end{array}$ & $<0.001$ & 1.097 & $\begin{array}{c}1.084- \\
1.111\end{array}$ & $<0.001$ & 1.097 & $\begin{array}{c}1.077- \\
1.117\end{array}$ & $<0.001$ & 1.104 & $\begin{array}{c}1.083- \\
1.126\end{array}$ & $<0.001$ \\
\hline Male & 2.291 & $\begin{array}{l}1.744- \\
3.008\end{array}$ & $<0.001$ & 2.785 & $\begin{array}{c}2.096- \\
3.702\end{array}$ & $<0.001$ & 1.968 & $\begin{array}{l}1.255- \\
3.087\end{array}$ & 0.003 & 2.404 & $\begin{array}{l}1.515- \\
3.815\end{array}$ & $<0.001$ \\
\hline $\begin{array}{l}\text { Body mass index, } \\
\mathrm{kg} / \mathrm{m}^{2}\end{array}$ & 1.063 & $\begin{array}{l}1.029- \\
1.098\end{array}$ & $<0.001$ & & & & 1.097 & $\begin{array}{l}1.041- \\
1.157\end{array}$ & 0.001 & & & \\
\hline $\begin{array}{l}\text { Waist circumference, } \\
\mathrm{cm}\end{array}$ & 1.032 & $\begin{array}{l}1.020- \\
1.044\end{array}$ & $<0.001$ & & & & 1.046 & $\begin{array}{l}1.026- \\
1.066\end{array}$ & $<0.001$ & & & \\
\hline Systolic BP, mmHg & 1.026 & $\begin{array}{l}1.019- \\
1.033\end{array}$ & $<0.001$ & & & & 1.027 & $\begin{array}{l}1.015- \\
1.039\end{array}$ & $<0.001$ & & & \\
\hline Diastolic BP, mmHg & 1.021 & $\begin{array}{l}1.011- \\
1.030\end{array}$ & $<0.001$ & & & & 1.022 & $\begin{array}{l}1.006- \\
1.038\end{array}$ & 0.007 & & & \\
\hline Diabetes mellitus & 2.479 & $\begin{array}{l}1.993- \\
3.085\end{array}$ & $<0.001$ & & & & 2.433 & $\begin{array}{l}1.697- \\
3.488\end{array}$ & $<0.001$ & & & \\
\hline Hypertension & 2.653 & $\begin{array}{l}2.175- \\
3.237\end{array}$ & $<0.001$ & & & & 2.950 & $\begin{array}{c}2.095- \\
4.154\end{array}$ & $<0.001$ & & & \\
\hline Hyperlipidemia & 1.785 & $\begin{array}{l}1.464- \\
2.177\end{array}$ & $<0.001$ & & & & 1.979 & $\begin{array}{l}1.419- \\
2.760\end{array}$ & $<0.001$ & & & \\
\hline Current smoker & 1.105 & $\begin{array}{c}0.883- \\
1.383\end{array}$ & 0.383 & & & & 1.107 & $\begin{array}{c}0.743- \\
1.648\end{array}$ & 0.617 & & & \\
\hline $\begin{array}{l}\text { Family history of } \\
\mathrm{CAD}^{+}\end{array}$ & 1.216 & $\begin{array}{c}0.941- \\
1.571\end{array}$ & 0.134 & 1.534 & $\begin{array}{l}1.172- \\
2.008\end{array}$ & 0.002 & 1.391 & $\begin{array}{l}0.919- \\
2.105\end{array}$ & 0.119 & 1.781 & $\begin{array}{l}1.160- \\
2.734\end{array}$ & 0.008 \\
\hline $\begin{array}{l}\text { Total cholesterol, } \\
\mathrm{mg} / \mathrm{dl}\end{array}$ & 1.001 & $\begin{array}{c}0.999- \\
1.004\end{array}$ & 0.565 & & & & 1.001 & $\begin{array}{l}0.996- \\
1.006\end{array}$ & 0.682 & & & \\
\hline $\begin{array}{l}\text { LDL-cholesterol, } \\
\mathrm{mg} / \mathrm{dl}\end{array}$ & 1.003 & $\begin{array}{c}0.999- \\
1.006\end{array}$ & 0.112 & 1.006 & $\begin{array}{c}1.003- \\
1.009\end{array}$ & $<0.001$ & 1.004 & $\begin{array}{c}0.999- \\
1.009\end{array}$ & 0.147 & 1.007 & $\begin{array}{l}1.002- \\
1.013\end{array}$ & 0.007 \\
\hline $\begin{array}{l}\text { HDL-cholesterol, } \\
\mathrm{mg} / \mathrm{dl}\end{array}$ & 0.974 & $\begin{array}{c}0.966- \\
0.982\end{array}$ & $<0.001$ & & & & 0.970 & $\begin{array}{c}0.956- \\
0.984\end{array}$ & $<0.001$ & & & \\
\hline Triglycerides, mg/dl & 1.002 & $\begin{array}{l}1.001- \\
1.003\end{array}$ & $<0.001$ & & & & 1.002 & $\begin{array}{l}1.000- \\
1.003\end{array}$ & 0.037 & & & \\
\hline $\begin{array}{l}\text { Fasting glucose, } \\
\mathrm{mg} / \mathrm{dl}\end{array}$ & 1.015 & $\begin{array}{l}1.012- \\
1.019\end{array}$ & $<0.001$ & & & & 1.011 & $\begin{array}{l}1.006- \\
1.016\end{array}$ & $<0.001$ & & & \\
\hline $\begin{array}{l}\text { Glycated hemoglobin, } \\
\%\end{array}$ & 1.634 & $\begin{array}{l}1.496- \\
1.785\end{array}$ & $<0.001$ & & & & 1.474 & $\begin{array}{l}1.306- \\
1.665\end{array}$ & $<0.001$ & & & \\
\hline Creatinine, mg/dl & 4.535 & $\begin{array}{l}2.476- \\
8.306\end{array}$ & $<0.001$ & & & & 5.038 & $\begin{array}{l}1.813- \\
14.000\end{array}$ & 0.002 & & & \\
\hline Uric acid, mg/dl & 1.127 & $\begin{array}{l}1.053- \\
1.207\end{array}$ & 0.001 & & & & 1.088 & $\begin{array}{c}0.969- \\
1.222\end{array}$ & 0.154 & & & \\
\hline $\mathrm{hsCRP} \geq 2 \mathrm{mg} / \mathrm{dl}$ & 1.420 & $\begin{array}{l}0.603- \\
3.344\end{array}$ & 0.422 & & & & 2.179 & $\begin{array}{l}0.671- \\
7.076\end{array}$ & 0.195 & & & \\
\hline $\begin{array}{l}\text { Framingham risk } \\
\text { score }\end{array}$ & 1.132 & $\begin{array}{l}1.113- \\
1.150\end{array}$ & $<0.001$ & & & & 1.117 & $\begin{array}{l}1.090- \\
1.146\end{array}$ & $<0.001$ & & & \\
\hline MetS & 2.211 & $\begin{array}{l}1.815- \\
2.694\end{array}$ & $<0.001$ & 1.992 & $\begin{array}{l}1.623- \\
2.445\end{array}$ & $<0.001$ & 2.431 & $\begin{array}{l}1.733- \\
3.411\end{array}$ & $<0.001$ & 2.151 & $\begin{array}{l}1.523- \\
3.037\end{array}$ & $<0.001$ \\
\hline
\end{tabular}

${ }^{*}$ Multiple logistic regression analysis (1) adjusting for age, male sex, family history of CAD, LDL-cholesterol, creatinine, uric acid, and MetS. ${ }^{* *}$ Multiple logistic regression analysis (2) adjusting for age, male sex, family history of CAD, LDL-cholesterol, creatinine, uric acid, hsCRP $\geq 2 \mathrm{mg} / \mathrm{dl}$, and MetS. ${ }^{+} \mathrm{CAD}$ in a first-degree relative of any age. $\mathrm{Cl}$, confidence interval; OR, odds ratio. Other abbreviations as in Tables 1,2 .

$\mathrm{P}<0.001)$. Furthermore, in the MetS with significant CAD group, those with significant CAD in the LM or proximal $\mathrm{LAD}$ had more cardiac events than those without $(\mathrm{RR}=2.63$, 95\% $\mathrm{CI}=1.51-4.59, \mathrm{P}=0.001)$.

We present a representative case. A 67-year-old man with MetS underwent a regular medical check-up including CCTA on July 9, 2008. On CCTA, significant stenosis was detected at the proximal LAD and first diagonal (D1) bifurcation lesion with dense calcified plaque (Figures $2 \mathrm{~A}-\mathrm{C}$ ) and a non-calcified plaque portion was revealed in the distal segment. At that time, we recommended further coronary evaluation, but he refused. Approximately 1 month later (August 11, 2008), he presented to the emergency room with resting chest pain.
Coronary angiography showed diffuse significant stenosis at the proximal to mid-LAD and D1 (Figure 2D). Therefore, we treated the patient by deploying 2 stents from the proximal to mid-LAD lesion and ballooning the D1 lesion (Figure 2E).

\section{Discussion}

The main findings of this study were as follows: (1) individuals with MetS had a higher prevalence of subclinical coronary atherosclerosis despite the absence of symptoms; (2) after adjustment for clinical risk factors, MetS was an independent predictor for significant CAD in at least 1 coronary artery and significant CAD in the LM or proximal LAD; (3) individuals 


\begin{tabular}{|c|c|c|c|c|c|c|c|c|c|}
\hline \multirow{3}{*}{ Overall population } & \multicolumn{6}{|c|}{ MetS } & \multirow[b]{3}{*}{ Rate ratio } & \multirow{4}{*}{$95 \% \mathrm{Cl}$} & \multirow[b]{3}{*}{$P$ value } \\
\hline & \multicolumn{3}{|c|}{ Yes $(n=2,042)$} & \multicolumn{3}{|c|}{ No $(n=3,171)$} & & & \\
\hline & $\mathbf{n}$ & Rate $^{*}$ & $95 \% \mathrm{Cl}$ & $\mathbf{n}$ & Rate $^{*}$ & $95 \% \mathrm{Cl}$ & & & \\
\hline \multicolumn{9}{|l|}{ Clinical outcome } & \\
\hline Death & 6 & 1.26 & $0.25-2.27$ & 13 & 1.75 & $0.80-2.70$ & 0.72 & $0.27-1.90$ & 0.510 \\
\hline Non-fatal MI & 1 & 0.21 & $0-0.62$ & 1 & 0.13 & $0-0.40$ & 1.57 & $0.10-25.05$ & 0.751 \\
\hline Unstable angina & 2 & 0.42 & $0-1.01$ & 0 & 0 & - & - & - & - \\
\hline Coronary revascularization & 50 & 10.78 & $7.79-13.77$ & 41 & 5.58 & $3.87-7.29$ & 1.93 & $1.28-2.92$ & 0.002 \\
\hline Death/MI/UA & 9 & 1.90 & $0.66-3.14$ & 14 & 1.88 & $0.90-2.87$ & 1.01 & $0.44-2.33$ & 0.984 \\
\hline \multirow[t]{2}{*}{ Death/MI/UA/revascularization } & 57 & 12.30 & $9.11-15.50$ & 54 & 7.35 & $5.39-9.31$ & 1.67 & $1.15-2.43$ & 0.007 \\
\hline & \multicolumn{6}{|c|}{ Significant CAD on CCTA } & & & \\
\hline \multirow[t]{2}{*}{ MetS group } & \multicolumn{3}{|c|}{ Yes $(n=251)$} & \multicolumn{3}{|c|}{ No $(n=1,791)$} & & & \\
\hline & $\mathbf{n}$ & Rate $^{*}$ & $95 \% \mathrm{Cl}$ & $\mathrm{n}$ & Rate $^{*}$ & $95 \% \mathrm{Cl}$ & Rate ratio & $95 \% \mathrm{Cl}$ & $P$ value \\
\hline \multicolumn{10}{|l|}{ Clinical outcome } \\
\hline Death/MI/UA/revascularization & 50 & 107.98 & $78.05-137.91$ & 7 & 1.68 & $0.44-2.92$ & 64.33 & $29.17-141.88$ & $<0.001$ \\
\hline \multicolumn{7}{|c|}{ Significant CAD in the LM or proximal LAD on CCTA } & & & \\
\hline $\begin{array}{l}\text { MetS with significant } \\
\text { CAD qroup }\end{array}$ & \multicolumn{3}{|c|}{ Yes $(n=87)$} & \multicolumn{3}{|c|}{ No $(n=164)$} & & & \\
\hline & $\mathbf{n}$ & Rate* $^{*}$ & $95 \% \mathrm{Cl}$ & $\mathbf{n}$ & Rate* $^{*}$ & $95 \% \mathrm{Cl}$ & Rate ratio & $95 \% \mathrm{Cl}$ & $P$ value \\
\hline \multicolumn{10}{|l|}{ Clinical outcome } \\
\hline Death/MI/UA/revascularization & 27 & 189.09 & $117.77-260.42$ & 23 & 71.82 & $42.47-101.17$ & 2.63 & $1.51-4.59$ & 0.001 \\
\hline
\end{tabular}

${ }^{*}$ Crude incidence rate per 1,000 person-years. MI, myocardial infarction; UA, unstable angina. Other abbreviations as in Tables 1-3.
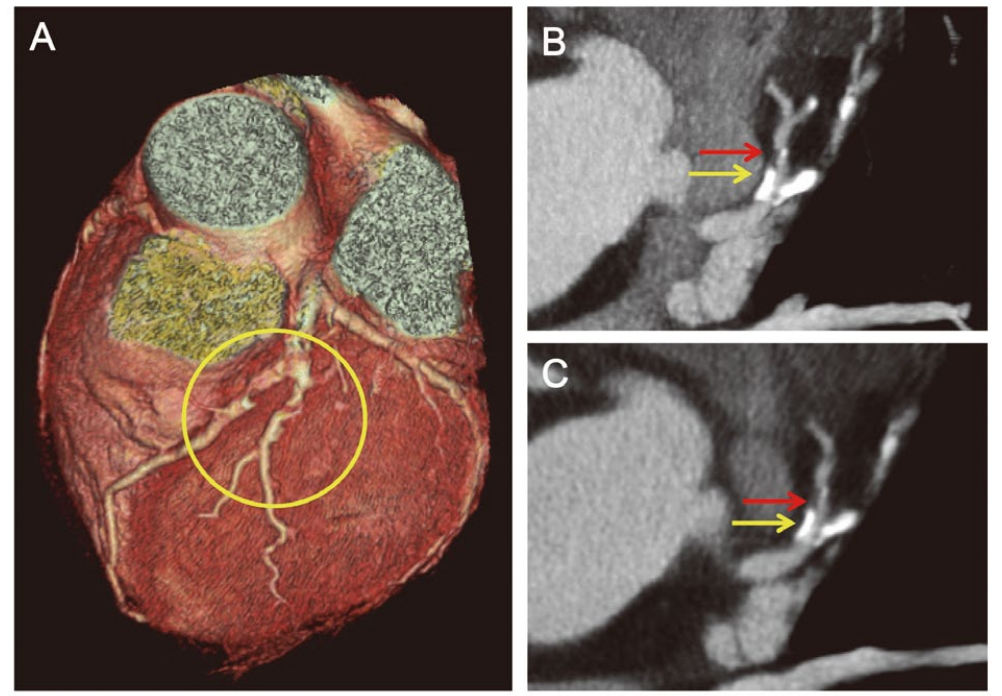

D
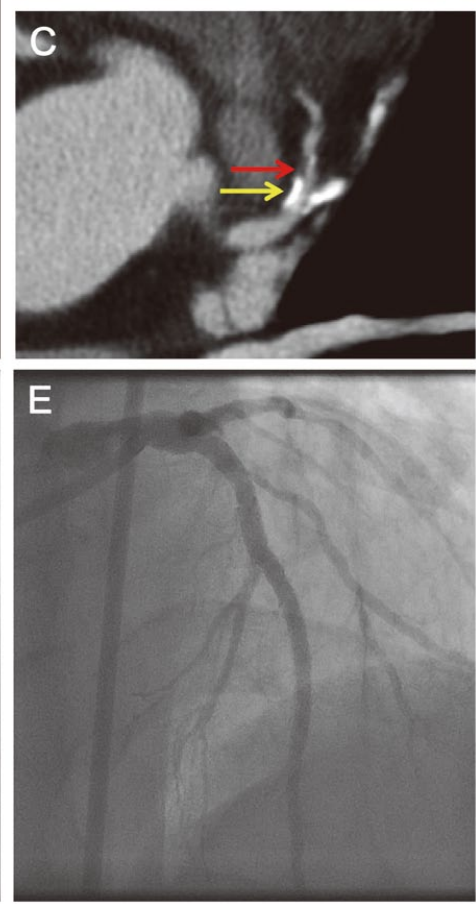

Figure 2. Coronary computed tomographic angiographic and invasive coronary angiographic findings in a 67-year-old male with metabolic syndrome presenting with acute coronary syndrome. (A) Volume-rendered image of first diagonal (D1) bifurcation lesion (yellow circle), (B) multiplanar reformation image and (C) maximum intensity projection image with dense calcified plaque (yellow arrow), and non-calcified plaque portion (red arrow) in the distal segment. (D) Coronary angiography shows diffuse significant stenosis at the proximal to mid-LAD and D1. (E) The patient was treated by deploying 2 stents from the proximal to mid-LAD lesion and ballooning the D1 lesion. 
with MetS had more cardiac events during the follow-up period than those without; and (4) in the MetS group, individuals with significant $\mathrm{CAD}$, and especially those with significant CAD involving the $\mathrm{LM}$ or proximal $\mathrm{LAD}$, had the majority of cardiac events.

The association between MetS and cardiovascular risk has been investigated in earlier studies, using carotid intima-media thickness, coronary artery calcification, and myocardial perfusion imaging. ${ }^{20-22}$ Recently, CCTA has been widely used in the comprehensive evaluation of $\mathrm{CAD}$, including lesion location, severity, and plaque characteristics. However, less is known about subclinical atherosclerosis assessed by CCTA in asymptomatic individuals with MetS. Previous observational studies using CCTA in this population showed that atherosclerotic plaque was observed in $30-52 \%$ and significant CAD occurred in $10 \%$ of individuals. ${ }^{7,9}$ Consistent with those studies, in our study, atherosclerotic plaques were identified in $1,101(53.9 \%)$ individuals, and of those, 251 (12.3\%) had significant stenosis on CCTA. In addition, after adjustment for clinical risk factors, MetS was associated with significant CAD in at least 1 coronary artery on CCTA. It is difficult to directly compare the findings of this study with those of previous studies because the age, sex, and associated comorbid conditions of the study groups were different. Nevertheless, in this and previous studies, individuals with MetS were associated with an approximately 2-fold increase in significant CAD on CCTA compared with those without. ${ }^{7}$ These findings imply that subclinical atherosclerosis in asymptomatic individuals with MetS is a problem that should not be ignored.

In this cohort, individuals with MetS had more multivessel disease and significant lesions in the LM or proximal LAD, which are known to be associated with a worse prognosis, compared with those without. ${ }^{17,23}$ Even after adjustment for other clinical risk factors, MetS was associated with significant CAD in the LM or proximal LAD on CCTA. Our findings are in agreement with a previous study in which asymptomatic individuals with MetS had more multivessel disease than those without. ${ }^{7}$ These findings indicate that MetS causes qualitatively as well as quantitatively worse subclinical atherosclerosis.

The mechanisms by which MetS increases cardiovascular risk are unclear. ${ }^{3}$ Our study provides some insights into the mechanisms. In this study, during the follow-up period, individuals with MetS had more cardiac events than those without. In the MetS group, the majority of cardiac events occurred in individuals with significant $\mathrm{CAD}$, especially in those with significant lesions in the LM or proximal LAD. These findings imply that the severity of subclinical coronary atherosclerosis determines subsequent cardiac events in asymptomatic individuals with MetS.

Cardiac events occur after long periods of subclinical disease. Early identification of individuals with subclinical atherosclerosis and aggressive lifestyle modification may prevent cardiac events in high-risk subjects. ${ }^{24}$ It is well known that MetS promotes or increases the risk of developing diabetes mellitus and subsequent cardiovascular disease. ${ }^{25}$ This and previous studies showed that subclinical atherosclerosis as assessed by CCTA was not negligible in asymptomatic individuals with MetS. ${ }^{7,9}$ The relationship between MetS and subclinical atherosclerosis is important from a prevention perspective. ${ }^{20}$ Furthermore, this study revealed that the severity of subclinical atherosclerosis determines future cardiac events in this population. Therefore, to prevent future cardiac events in asymptomatic individuals, efforts should be made to reduce the unfavorable metabolic conditions.

\section{Study Limitations}

First, the present study was performed in a single center, so there was potential for selection bias. Second, the study population was exclusively Korean, which limits the applicability of our findings to other ethnic groups. Third, calcified plaques and higher CACS may lead to overestimation of significant CAD. ${ }^{26}$ Fourth, most of cardiac events were coronary revascularizations, which is partly attributable to the fact that all individuals went to hospital for their general health examination and were asymptomatic. Therefore, in asymptomatic individuals with MetS, future studies to evaluate the association between hard clinical events and subclinical atherosclerosis are needed. Fifth, the napkin-ring sign, positive vessel remodeling, and low-attenuation plaque are known as high-risk plaque features. ${ }^{27,28}$ In our cohort, the number of individuals with acute coronary syndrome (MI and unstable angina) was only $4(0.08 \%)$ and analysis of these characteristics was not performed. Other large studies are required to elucidate the clinical effect of these high-risk plaque characteristics in asymptomatic individuals. Finally, CCTA itself has potential limitations, including radiation hazard, use of contrast, and higher cost. ${ }^{29}$ Although our study enrolled only volunteers, the use of CCTA in asymptomatic individuals with MetS has not yet been justified.

\section{Conclusions}

This large observational study of asymptomatic individuals with MetS showed that the prevalence of subclinical atherosclerosis was not negligible. Individuals with significant CAD, and particularly those with significant CAD in the LM or proximal LAD, had a subsequent risk of cardiac events. For the prevention of cardiac events in asymptomatic individuals, our results suggest the importance of reducing unfavorable metabolic conditions.

\section{Conflict of Interest}

The authors have no conflicts of interest to declare.

\section{Acknowledgments}

This study was supported by a grant from the Korea Healthcare Technology R\&D Project, Ministry of Health and Welfare, Republic of Korea (A102065, HI12C0630, and HI10C2020).

\section{References}

1. Grundy SM. Metabolic syndrome pandemic. Arterioscler Thromb Vasc Biol 2008; 28: 629-636.

2. Tajima M, Lee JS, Watanabe E, Park JS, Tsuchiya R, Fukahori A, et al. Association between changes in 12 lifestyle behaviors and the development of metabolic syndrome during 1 year among workers in the Tokyo metropolitan area. Circ J 2014; 78: $1152-1159$.

3. Mottillo S, Filion KB, Genest J, Joseph L, Pilote L, Poirier P, et al. The metabolic syndrome and cardiovascular risk a systematic review and meta-analysis. J Am Coll Cardiol 2010; 56: 1113-1132.

4. Nakanishi K, Nishida M, Ohama T, Moriyama T, Yamauchi-Takihara $\mathrm{K}$. Smoking associates with visceral fat accumulation especially in women. Circ J 2014; 78: 1259-1263.

5. Matsuzawa Y, Sugiyama S, Sugamura K, Sumida H, Kurokawa H, Fujisue K, et al. Successful diet and exercise therapy as evaluated on self-assessment score significantly improves endothelial function in metabolic syndrome patients. Circ J 2013; 77: 2807-2815.

6. Empana JP, Duciemetiere P, Balkau B, Jouven X. Contribution of the metabolic syndrome to sudden death risk in asymptomatic men: The Paris Prospective Study I. Eur Heart J 2007; 28: 1149-1154.

7. Lim S, Shin H, Lee Y, Yoon JW, Kang SM, Choi SH, et al. Effect of metabolic syndrome on coronary artery stenosis and plaque characteristics as assessed with 64-detector row cardiac CT. Radiology 2011; 261: 437-445.

8. Park GM, Lee SW, Cho YR, Kim CJ, Cho JS, Park MW, et al. 
Coronary computed tomographic angiographic findings in asymptomatic patients with type 2 diabetes mellitus. Am J Cardiol 2014; 113: $765-771$

9. Ryu J, Yong HS, Huh S, Kang EY, Woo OH. Relation of coronary atherosclerosis and metabolic syndrome in asymptomatic subjects: Evaluation with coronary CT angiography. Int J Cardiovasc Imaging 2013; 29(Suppl 2): $101-107$.

10. Wilson PW, D'Agostino RB, Levy D, Belanger AM, Silbershatz H, Kannel WB. Prediction of coronary heart disease using risk factor categories. Circulation 1998; 97: 1837-1847.

11. Grundy SM, Cleeman JI, Daniels SR, Donato KA, Eckel RH, Franklin $\mathrm{BA}$, et al. Diagnosis and management of the metabolic syndrome: An American Heart Association/National Heart, Lung, and Blood Institute Scientific Statement. Circulation 2005; 112: 2735-2752.

12. Raff GL, Abidov A, Achenbach S, Berman DS, Boxt LM, Budoff MJ, et al. SCCT guidelines for the interpretation and reporting of coronary computed tomographic angiography. J Cardiovasc Comput Tomogr 2009; 3: 122-136.

13. Agatston AS, Janowitz WR, Hildner FJ, Zusmer NR, Viamonte M $\mathrm{Jr}$, Detrano R. Quantification of coronary artery calcium using ultrafast computed tomography. J Am Coll Cardiol 1990; 15: 827-832.

14. Rumberger JA, Brundage BH, Rader DJ, Kondos G. Electron beam computed tomographic coronary calcium scanning: A review and guidelines for use in asymptomatic persons. Mayo Clin Proc 1999; 74: $243-252$

15. Leber AW, Becker A, Knez A, von Ziegler F, Sirol M, Nikolaou K, et al. Accuracy of 64-slice computed tomography to classify and quantify plaque volumes in the proximal coronary system: A comparative study using intravascular ultrasound. J Am Coll Cardiol 2006; 47: 672-677.

16. Hausleiter J, Meyer T, Hadamitzky M, Kastrati A, Martinoff S, Schomig A. Prevalence of noncalcified coronary plaques by 64 -slice computed tomography in patients with an intermediate risk for significant coronary artery disease. J Am Coll Cardiol 2006; 48: 312318.

17. Min JK, Shaw LJ, Devereux RB, Okin PM, Weinsaft JW, Russo DJ, et al. Prognostic value of multidetector coronary computed tomographic angiography for prediction of all-cause mortality. $J \mathrm{Am} \mathrm{Coll}$ Cardiol 2007; 50: 1161-1170.

18. Jee SH, Sull JW, Park J, Lee SY, Ohrr H, Guallar E, et al. Body-mass index and mortality in Korean men and women. N Engl J Med 2006 355: $779-787$.

19. Group BDS, Frye RL, August P, Brooks MM, Hardison RM, Kelsey
SF, et al. A randomized trial of therapies for type 2 diabetes and coronary artery disease. $N$ Engl J Med 2009; 360: 2503-2515.

20. Ahluwalia N, Drouet L, Ruidavets JB, Perret B, Amar J, Boccalon $\mathrm{H}$, et al. Metabolic syndrome is associated with markers of subclinical atherosclerosis in a French population-based sample. Atherosclerosis 2006; 186: $345-353$.

21. Chen K, Lindsey JB, Khera A, De Lemos JA, Ayers CR, Goyal A, et al. Independent associations between metabolic syndrome, diabetes mellitus and atherosclerosis: Observations from the Dallas Heart Study. Diab Vasc Dis Res 2008; 5: 96-101.

22. Wong ND, Rozanski A, Gransar H, Miranda-Peats R, Kang X, Hayes S, et al. Metabolic syndrome and diabetes are associated with an increased likelihood of inducible myocardial ischemia among patients with subclinical atherosclerosis. Diabetes Care 2005; 28: $1445-1450$.

23. Pundziute G, Schuijf JD, Jukema JW, Boersma E, de Roos A, van der Wall EE, et al. Prognostic value of multislice computed tomography coronary angiography in patients with known or suspected coronary artery disease. J Am Coll Cardiol 2007; 49: 62-70.

24. Malik S, Wong ND. Metabolic syndrome, cardiovascular risk and screening for subclinical atherosclerosis. Expert Rev Cardiovasc Ther 2009; 7: 273-280.

25. Ford ES, Li C, Sattar N. Metabolic syndrome and incident diabetes: Current state of the evidence. Diabetes Care 2008; 31: 1898-1904.

26. Arbab-Zadeh A, Miller JM, Rochitte CE, Dewey M, Niinuma H, Gottlieb I, et al. Diagnostic accuracy of computed tomography coronary angiography according to pre-test probability of coronary artery disease and severity of coronary arterial calcification: The CORE-64 (Coronary Artery Evaluation Using 64-Row Multidetector Computed Tomography Angiography) International Multicenter Study. $J$ Am Coll Cardiol 2012; 59: 379-387.

27. Maurovich-Horvat $P$, Hoffmann U, Vorpahl M, Nakano M, Virmani $\mathrm{R}$, Alkadhi $\mathrm{H}$. The napkin-ring sign: CT signature of high-risk coronary plaques? JACC Cardiovasc Imaging 2010; 3: 440-444.

28. Motoyama S, Sarai M, Harigaya H, Anno H, Inoue K, Hara T, et al. Computed tomographic angiography characteristics of atherosclerotic plaques subsequently resulting in acute coronary syndrome. $J$ Am Coll Cardiol 2009; 54: 49-57.

29. Cho I, Shim J, Chang HJ, Sung JM, Hong Y, Shim H, et al. Prognostic value of multidetector coronary computed tomography angiography in relation to exercise electrocardiogram in patients with suspected coronary artery disease. J Am Coll Cardiol 2012; 60: 2205-2215. 